\title{
Article \\ Genetic Variants in Smoking-Related Genes in Two Smoking Cessation Programs: A Cross-Sectional Study
}

\author{
Gloria Pérez-Rubio ${ }^{1}$ D, Luis Alberto López-Flores ${ }^{1}$ (D), Ana Paula Cupertino ${ }^{2}$, Francisco Cartujano-Barrera ${ }^{2}{ }^{\mathbb{D}}$, \\ Luz Myriam Reynales-Shigematsu ${ }^{3}$, Mariana Ramírez ${ }^{4}$, Edward F. Ellerbeck ${ }^{4}$, Rosibel Rodríguez-Bolaños ${ }^{3}$ i \\ and Ramcés Falfan-Valencia $1, *$ (D)
}

check for

updates

Citation: Pérez-Rubio, G.;

López-Flores, L.A.; Cupertino, A.P.;

Cartujano-Barrera, F.; Reynales-

Shigematsu, L.M.; Ramírez, M.

Ellerbeck, E.F.; Rodríguez-Bolaños, R.; Falfan-Valencia, R. Genetic Variants in Smoking-Related Genes in Two Smoking Cessation Programs: A

Cross-Sectional Study. Int. J. Environ. Res. Public Health 2021, 18, 6597. https://doi.org/10.3390/ ijerph18126597

Academic Editors: Ashley

L. Merianos and E.

Melinda Mahabee-Gittens

Received: 15 April 2021

Accepted: 16 June 2021

Published: 19 June 2021

Publisher's Note: MDPI stays neutral with regard to jurisdictional claims in published maps and institutional affiliations.

Copyright: (c) 2021 by the authors. Licensee MDPI, Basel, Switzerland. This article is an open access article distributed under the terms and conditions of the Creative Commons Attribution (CC BY) license (https:// creativecommons.org/licenses/by/ $4.0 /)$
1 HLA Laboratory, Instituto Nacional de Enfermedades Respiratorias Ismael Cosío Villegas, Calzada de Tlalpan 4502, Sección XVI, Mexico City 14080, Mexico; glofos@yahoo.com.mx (G.P.-R.); llopezf92@gmail.com (L.A.L.-F.)

2 Department of Cancer Prevention and Control, Hackensack University Medical Center, Hackensack, NJ 07601, USA; paula.cupertino@hackensackmeridian.org (A.P.C.); francisco.cartujano@hackensackmeridian.org (F.C.-B.)

3 Department of Tobacco Research, Instituto Nacional de Salud Pública, Cuernavaca 62100, Mexico; lreynales@insp.mx (L.M.R.-S.); rrodriguez@insp.mx (R.R.-B.)

4 Department of Preventive Medicine and Public Health, University of Kansas Medical Center, Kansas City, KS 66160, USA; mramirez3@kumc.edu (M.R.); eellerbe@kumc.edu (E.F.E.)

* Correspondence: rfalfanv@iner.gob.mx; Tel.: +52-55-5487-1700 (ext. 5152)

\begin{abstract}
Previous studies have identified variants in genes encoding proteins associated with the degree of addiction, smoking onset, and cessation. We aimed to describe thirty-one single nucleotide polymorphisms (SNPs) in seven candidate genomic regions spanning six genes associated with tobacco-smoking in a cross-sectional study from two different interventions for quitting smoking: (1) thirty-eight smokers were recruited via multimedia to participate in e-Decídete! program (e-Dec) and (2) ninety-four attended an institutional smoking cessation program on-site. SNPs genotyping was done by real-time PCR using TaqMan probes. The analysis of alleles and genotypes was carried out using the EpiInfo v7. on-site subjects had more years smoking and tobacco index than e-Dec smokers ( $p<0.05$, both); in CYP2A6 we found differences in the rs28399433 ( $p<0.01)$, the e-Dec group had a higher frequency of TT genotype ( 0.78 vs. 0.35$)$, and TG genotype frequency was higher in the on-site group (0.63 vs. 0.18 ), same as GG genotype (0.03 vs. 0.02$)$. Moreover, three SNPs in NRXN1, two in CHRNA3, and two in CHRNA5 had differences in genotype frequencies $(p<0.01)$. Cigarettes per day were different $(p<0.05)$ in the metabolizer classification by CYP2A6 alleles. In conclusion, subjects attending a mobile smoking cessation intervention smoked fewer cigarettes per day, by fewer years, and by fewer cumulative pack-years. There were differences in the genotype frequencies of SNPs in genes related to nicotine metabolism and nicotine dependence. Slow metabolizers smoked more cigarettes per day than intermediate and normal metabolizers.
\end{abstract}

Keywords: nicotine addiction; smoking cessation; CHRNA3; CHRNA5; NRXN1; DRD4; HTR2A; CYP2A6; e-Decídete

\section{Introduction}

Cigarette consumption is a global public health problem. In Mexico, around 60,000 people die a year (between 165 and 180 deaths a day) due to diseases associated with tobacco use, according to the statistics from the General Directorate of Epidemiology of the Ministry of Health. According to the latest National Survey on Drug, Alcohol, and Tobacco Consumption (ENCODAT) 2016-2017 [1], there are more than 15.6 million smokers in Mexico, and the most vulnerable group is young people between 12 and 15 years of age. Both men and women smoke tobacco alike [1]. In addition, the Global Adult Tobacco Survey 2015 (GATS) reports that $17.6 \%$ of the population between 12-65 years old smoke tobacco; of these, $73.6 \%$ are interested in quitting smoking, $56.1 \%$ have tried to quit such drug, but only 
$1.2 \%$ underwent some treatment to leave; only $3.5 \%$ used pharmacotherapy, $5.9 \%$ used counseling, and $90.6 \%$ used willpower [2]. Furthermore, it is unclear whether subjects with different smoking behavior characteristics can access mobile smoking cessation services. Tobacco smoking is a common and multifactorial disease where the environmental and genetic factors participate in a complex network where gene products are involved in addictive pathways [3]. Genome-wide association studies (GWAS) have identified some loci with smoking patterns of cigarette consumption in nicotinic cholinergic receptors genes (CHRNA5-CHRNA3-CHRNB4) [4], neurotransmitter receptors' genes (HTR2A, DRD4, etc.), as well as those involved in the metabolism of nicotine such as CYP2A6, which is a liver enzyme that metabolizes between $70 \%-80 \%$ of the nicotine that enters the body in cotinine [5]. At the genetic level, it has been reported that there are more than 30 allelic variants that affect the enzymatic activity [6,7]. The CYP2A6*1A allele is considered as the reference, while the presence of $C Y P 2 A 6^{*} 1 \mathrm{~B}$ causes low expression of the mRNA, in vitro studies have shown a low rate of nicotine metabolism [8]. Several alleles have been described as decreased enzymatic function, and mainly these alleles are produced by SNPs (CYP2A6*5, *7, *9, *18, *21, *23*25, *28, and *35 [9]). Several GWAS performed in Asian, European, and African populations identified polymorphisms in the CHRNA5 and CYP2A6 genes strongly associated with the risk of smoking [10], being a heavy smoker ( $\geq 20$ cigarettes per day) [11], to a better success in the treatment to stop smoking [9] or in the development of diseases caused by cigarette smoking (mainly COPD and lung cancer) $[12,13]$.

We aimed to describe smoking behavior, allele, and genotype frequencies of polymorphic variants in six candidate genes in a population of smokers recruited by a mobile smoking cessation intervention and compare to people attending an institutional smokingcessation program.

\section{Materials and Methods}

\subsection{Population Study}

A cross-sectional study was performed with baseline data from two different interventions for quitting smoking. (1) e-Decídete! program daily smokers and (2) daily smokers attending an institutional smoking cessation program on-site were included.

e-Decídete! program: this study aimed to assess the feasibility and acceptability of an innovative, personalized, and interactive smoking cessation mobile intervention developed for Mexican smokers. Participants were recruited through printed posters and multimedia venues, including ads through the National Institute of Public Health (Instituto Nacional de Salud Pública, Morelos, Mexico) website, Facebook, and local radio announcements. Potential participants emailed or called study personnel to learn more about the study. An eligibility assessment was conducted over the phone. Eligible participants were of Mexican origin, $\geq 18$ years of age, had smoked for at least 6 months, smoked at least 3 days per week, interested in quitting within the next 30 days, had a cellphone with text messaging capacity, and were willing to complete baseline and 12-week follow-up surveys. Participants were excluded from the study if they were planning to move within the next six months, consumed other forms of tobacco (including e-cigarettes), or had another household member enrolled in the study. All subjects gave informed consent prior to participation in the study. Participants received $300 \mathrm{MXN}(\approx 15 \mathrm{USD})$ at baseline and follow-up as an incentive for their time and transportation. This study was conducted between March and August 2017 at the Medical Center of the Autonomous University of the State of Morelos, located in Cuernavaca, Morelos, Mexico.

Smokers attending smoking cessation program on-site: participants were recruited from a quit-smoking help clinic from the Department of Research in Smoking and COPD of the National Institute of Respiratory Diseases Ismael Cosío Villegas (Instituto Nacional de Enfermedades Respiratorias Ismael Cosío Villegas (INER)) of Mexico. Only Mexican Mestizos by ancestry (Mexican-birth parents and grandparents) were included. Individuals with bronchial asthma, COPD, bronchiectasis, active tuberculosis, lung cancer, cystic fibrosis, hypersensitivity pneumonitis, or idiopathic pulmonary fibrosis were excluded 
from the study. To reduce difference among main covariates and avoid confounding factors, we matched intentionally this sample group by age and cigarettes per day.

The Human Subjects Committees of the Mexican National Institute of Public Health (INSP) and the Respiratory Diseases National Institute Ismael Cosio Villegas (INER, protocol numbers B07-17 and B15-16) approved the study procedures. The participants were invited to participate in the present research study and were informed about its aim. The participants then signed a letter of informed consent and were provided with an assuranceof-personal-data document. The Research Institute's Committee approved both documents of Science and Bioethical Research. Each participant was assigned an alphanumeric key to assure confidentiality.

\subsection{Samples Obtention}

An 8-mL volume of peripheral blood was obtained using venipuncture techniques and collected in a tube with EDTA as an anticoagulant for subsequent DNA extraction using a BDtract DNA Isolation Kit (Maxim Biotech, San Francisco, CA, USA). The DNA was quantified by ultraviolet absorption at a 260-nm wavelength using a NanoDrop instrument (Thermo Scientific, Waltham, MA, USA). Contamination with organic compounds and proteins was determined by establishing the relationship between the 260/240 and $260 / 280$ readings, respectively. The samples were considered free of contaminants when the relationship was found to be between 1.7 and 2.0.

\subsection{Single Nucleotide Variation Selection}

Two types of polymorphisms were evaluated: those SNPs forming CYP2A6 alleles involved in enzymatic activity alterations and a set of polymorphisms previously found associated with smoking and/or its behavior in a Mexican mestizo population.

SNPs in CYP2A6 were selected according to their allele assignation by the Pharmacogene Variation Consortium [14]. Minor alleles from these SNPs could be in more than an allele, whereby we select a specific set of SNPs that could discriminate a particular allele; therefore, each SNP (or set of SNPs) corresponds to a single allele. Fourteen polymorphisms at the CYP2A6 gene were included, of which 10 are missense, 2 synonymous, one $2 \mathrm{~KB}$ upstream, and one intron variant, spanning $6647 \mathrm{bp}$ at q13.2 in chromosome 19. Next, we search for the enzyme's main effect by carrying each allele in each subject genotype and then classify it as a type of normal, intermediate, or slow metabolizer. Table 1 collects information about molecular characteristics and frequencies for SNPs in CYP2A6 related to the enzymatic activity. 
Table 1. Molecular characteristics and frequencies for SNPs in CYP2A6 related to the enzymatic activity.

\begin{tabular}{|c|c|c|c|c|c|c|}
\hline \multirow{2}{*}{ SNP } & \multirow{2}{*}{ Chr Position } & \multirow{2}{*}{$\begin{array}{l}\text { Allele } \\
\text { Change }\end{array}$} & \multirow{2}{*}{$\begin{array}{c}\text { MAF } \\
\text { (GnomAD) }\end{array}$} & \multirow{2}{*}{$\begin{array}{l}\text { Consequence/Gene } \\
\text { Location }\end{array}$} & \multicolumn{2}{|c|}{ Residue } \\
\hline & & & & & Change & Position \\
\hline rs28399433 & chr19:40850474 & $A>C$ & $C=0.10323$ & 2KB Upstream Variant & NA & NA \\
\hline rs28399434 & chr19:40850414 & $\mathrm{C}>\mathrm{T}$ & $\mathrm{T}=0.00003$ & Missense Variant & G [Gly] $\Rightarrow$ R [Arg] & 5 \\
\hline rs 8192720 & chr19:40850405 & $G>A$ & $\mathrm{~A}=0.02214$ & Synonymous Variant & $\mathrm{L}[\mathrm{Leu}] \Rightarrow \mathrm{L}[\mathrm{Leu}]$ & 8 \\
\hline rs1137115 & chr19:40850376 & $\mathrm{T}>\mathrm{C}$ & $\mathrm{T}=0.24442$ & Synonymous Variant & $\mathrm{V}[\mathrm{Val}] \Rightarrow \mathrm{V}[\mathrm{Val}]$ & 17 \\
\hline rs1801272 & chr19:40848628 & $\mathrm{A}>\mathrm{T}$ & $\mathrm{T}=0.01974$ & Missense Variant & $\mathrm{L}[\mathrm{Leu}] \Rightarrow \mathrm{H}[\mathrm{His}]$ & 160 \\
\hline rs199916117 & chr19:40848293 & $\mathrm{T}>\mathrm{C}$ & $C=0.00058$ & Missense Variant & $\mathrm{K}$ [Lys] $\Rightarrow \mathrm{E}[\mathrm{Glu}]$ & 194 \\
\hline rs148166815 & chr19:40845404 & $A>G$ & $G=0.00008$ & Missense Variant & $\mathrm{Y}[\mathrm{Tyr}] \Rightarrow \mathrm{H}[\mathrm{His}]$ & 351 \\
\hline rs1809810 & chr19:40844759 & $\mathrm{T}>\mathrm{A}$ & $A=0.01259$ & Missense Variant & $\mathrm{Y}[\mathrm{Tyr}] \Rightarrow \mathrm{F}$ [Phe] & 392 \\
\hline rs2431413 & chr19:40844073 & $A>G$ & $\mathrm{G}=0.12103$ & Intron Variant & NA & NA \\
\hline rs143731390 & chr19:40843969 & $\mathrm{T}>\mathrm{A}$ & $\mathrm{A}=0.1020$ & Missense Variant & $\mathrm{N}[$ Asn $] \Rightarrow \mathrm{Y}[\mathrm{Tyr}]$ & 438 \\
\hline rs5031016 & chr19:40843869 & $A>G$ & $\mathrm{G}=0.00775$ & Missense Variant & $\mathrm{I}[$ Ile] $\Rightarrow \mathrm{T}$ [Thr] & 471 \\
\hline rs6413474 & chr19:40843854 & $\mathrm{T}>\mathrm{C}$ & $C=0.01235$ & Missense Variant & $\mathrm{K}[$ Lys $] \Rightarrow \mathrm{R}[\mathrm{Arg}]$ & 476 \\
\hline rs5031017 & chr19:40843845 & $\mathrm{C}>\mathrm{A}$ & $\mathrm{A}=0.00049$ & Missense Variant & G [Gly] $\Rightarrow$ V [Val] & 479 \\
\hline rs28399468 & chr19:40843827 & $\mathrm{C}>\mathrm{A}$ & $A=0.00165$ & Missense Variant & $\mathrm{R}[$ Arg $] \Rightarrow \mathrm{P}[$ Pro $]$ & 485 \\
\hline
\end{tabular}

SNP: single nucleotide polymorphism; Chr: chromosome; MAF: minor allele frequency; GnomAD: genome aggregation database; NA: not apply.

Moreover, a set of seventeen polymorphisms previously reported on the fine mapping of six loci were evaluated; these include 12 intron variants, 1 missense (CHRNA5: rs16969968, [Asp398Asn]), two 2KB-upstream, one synonymous (HTR2A: rs6313, [Ser34Ser]) and one intergenic variant. These 17 SNPs were selected because they were associated with cigarette smoking or a high degree of nicotine addiction in Mexican mestizo smokers. $[15,16]$. Characteristics of evaluated SNPs are disposable in Table 2.

Table 2. Characteristics of polymorphisms previously reported on the fine mapping of six genomic regions.

\begin{tabular}{|c|c|c|c|c|c|}
\hline SNP & Gene & Chr Position & Allele Change & MAF (GnomAD) & $\begin{array}{c}\text { Consequence/Gene } \\
\text { Location }\end{array}$ \\
\hline rs10189159 & \multirow{4}{*}{ NRXN1 } & chr2:50487433 & $\mathrm{T}>\mathrm{C}$ & $C=0.3286$ & Intron Variant \\
\hline rs985919 & & chr2:50459875 & $C>A$ & $\mathrm{~A}=0.4957$ & Intron Variant \\
\hline rs10865246 & & chr2:50443116 & $C>A$ & $C=0.4950$ & Intron Variant \\
\hline rs1882296 & & chr2:50039707 & $\mathrm{T}>\mathrm{C}$ & $C=0.3900$ & Intron Variant \\
\hline rs1800955 & DRD4 & chr11:636784 & $\mathrm{T}>\mathrm{C}$ & $C=0.406$ & 2KB Upstream Variant \\
\hline rs6311 & \multirow[b]{2}{*}{ HTR2A } & chr13:46897343 & $\mathrm{C}>\mathrm{T}$ & $\mathrm{T}=0.3970$ & 2KB Upstream Variant \\
\hline rs6313 & & chr13:46895805 & $G>A$ & $\mathrm{~A}=0.40701$ & Synonymous Variant \\
\hline rs12914385 & \multirow{4}{*}{ CHRNA3 } & chr15:78606381 & $C>A$ & $\mathrm{~T}=0.3138$ & Intron Variant \\
\hline rs1317286 & & chr15:78603787 & $A>G$ & $G=0.2974$ & Intron Variant \\
\hline rs6495307 & & chr15:78597979 & $\mathrm{C}>\mathrm{T}$ & $\mathrm{T}=0.310$ & Intron Variant \\
\hline rs615470 & & chr15:78593646 & $\mathrm{T}>\mathrm{C}$ & $\mathrm{T}=0.3688$ & Intron Variant \\
\hline rs16969968 & \multirow{5}{*}{ CHRNA5 } & chr15:78590583 & $G>A$ & $A=0.26553$ & Missense Variant \\
\hline rs17408276 & & chr15:78589276 & $\mathrm{T}>\mathrm{C}$ & $C=0.2881$ & Intron Variant \\
\hline rs951266 & & chr15:78586199 & $\mathrm{G}>\mathrm{A}$ & $\mathrm{A}=0.2522$ & Intron Variant \\
\hline rs680244 & & chr15:78578946 & $\mathrm{T}>\mathrm{C}$ & $\mathrm{T}=0.4098$ & Intron Variant \\
\hline rs17486278 & & chr15:78575140 & $A>C$ & $C=0.3167$ & Intron Variant \\
\hline rs4105144 & CYP2A6 near & chr19:40852719 & $\mathrm{T}>\mathrm{C}$ & $C=0.4412$ & Intergene \\
\hline
\end{tabular}

SNP: single nucleotide polymorphism; Chr: chromosome; MAF: minor allele frequency; GnomAD: genome aggregation database; NA: not apply.

\subsection{Genotyping}

Genotyping was performed using TaqMan allelic discrimination real-time PCR with pre-designed probes and a real-time PCR thermocycler (7300 Real-Time PCR Systems, Applied Biosystems/Thermo Fisher Scientific Inc., Singapore); considering the allelic 
discrimination and confirmed by absolute quantitation; three controls without template (contamination controls) were included for each genotyping plate, and $5 \%$ of the samples included in the study were genotyped in duplicate as controls for allele assignment. Data interpretation was conducted using Sequence Detection Software (SDS v. 1.4, Applied Biosystems, Waltham, MA, USA). VIC and FAM fluorophores were used for alleles A and $B$, respectively.

\subsection{Statistical Analysis}

The software SPSS v.20.0 was used (SPSS software, IBM, New York, NY, USA) to determine each variable's median and interquartile range. For the allele and genotype frequencies were employed Epidat version 3.1 software. [17]. For comparisons between genotype frequencies, Pearson's $\chi^{2}$ test was employed when they had individuals in three genotypes in one group and at least two genotypes in the other. Otherwise, we use the Fisher exact test when both groups had only two genotypes each.

\section{Results}

The Vive sin Tabaco ... jDecídete!: Single-Arm Pilot Study is a mobile smoking cessation intervention previously described [18].

\subsection{Study Population}

Thirty-eight smokers attending the e-Decídete! program (e-Dec) were included, while smokers attending an institutional smoking cessation program (on-site) were ninety-four. The demographic variables for both study groups are presented in Table 3 . This shows the median and minimum, and maximum values in each variable.

Table 3. Demographical variables.

\begin{tabular}{cccc}
\hline Variable & e-Dec $(\boldsymbol{n}=\mathbf{3 8})$ & On-Site $(\boldsymbol{n}=\mathbf{9 4 )}$ & $\boldsymbol{p}^{*}$ \\
\hline Age (years) & $35(20-60)$ & $36(20-40)$ & 0.700 \\
Male/Female $n,(\%)$ & $24 / 14(63.2 / 36.8)$ & $57 / 37(60.6 / 39.4)$ & 0.942 \\
Cigarettes per day & $9(2-30)$ & $10(1-60)$ & 0.064 \\
Years of smoking & $12(2-44)$ & $16(1-43)$ & 0.049 \\
Onset smoking age & $20.5(12-50)$ & $17.5(9-50)$ & 0.004 \\
Tobacco index & $6(0.6-66.0)$ & $9.8(0.1-38.7)$ & 0.015 \\
\hline
\end{tabular}

Median (minimum and maximum values). ${ }^{*} p$-value with Mann-Whitney $\mathrm{U}$ test.

\subsection{Genotype Frequencies}

Of the 15 SNPs located in CYP2A6, only 6 (rs28399433, rs28399434, rs8192720, rs1137115, rs2431413, and rs5031017) were polymorphic in at least two genotypes in a group. Comparing genotype frequencies between both groups, we find differences in the rs28399433 $(p<0.0001)$, in the e-Dec group there was a higher frequency of TT genotype $(0.78 \mathrm{vs} .0 .35)$, and TG genotype frequency was higher in the on-site group (0.63 vs. 0.18), same as GG genotype (0.03 vs. 0.02). Moreover, in rs2431413, genotype frequencies between both groups were different, in which only the on-site group had variation in frequency. Otherwise, rs1137115 $(p=0.768)$ and rs5031017 $(p=0.700)$ had similar genotype frequencies with non-significant statistical $p$-values; these data are shown in Table 4. Due to the lack of minor allele homozygous genotype individuals, we compare frequencies of major allele homozygous versus heterozygous genotypes in rs28399434 $(p=0.181)$ and rs8192720 $(p=0.287)$, with no statistically significant differences. 
Table 4. Genotype and allele frequencies in both groups included in this study only in six SNPs of CYP2A6.

\begin{tabular}{|c|c|c|c|c|c|}
\hline \multirow{2}{*}{$\begin{array}{c}\text { SNP } \\
\text { Genotype/Allele }\end{array}$} & \multicolumn{2}{|c|}{ e-Dec } & \multicolumn{2}{|c|}{ On-Site } & \multirow{2}{*}{$p^{*}$} \\
\hline & $n=38$ & GF/AF \% & $n=94$ & GF/AF \% & \\
\hline \multicolumn{6}{|l|}{ rs28399433 } \\
\hline $\mathrm{TT}$ & 30 & 78.95 & 32 & 34.04 & \multirow{3}{*}{$<0.0001$} \\
\hline TG & 7 & 18.42 & 59 & 62.77 & \\
\hline GG & 1 & 2.63 & 3 & 3.19 & \\
\hline $\mathrm{T}$ & 67 & 88.16 & 123 & 65.43 & \multirow[b]{2}{*}{0.0003} \\
\hline G & 9 & 11.84 & 65 & 34.57 & \\
\hline \multicolumn{6}{|l|}{ rs28399434 } \\
\hline GG & 38 & 100.00 & 88 & 93.62 & \multirow{3}{*}{ NS } \\
\hline GA & 0 & 0.00 & 6 & 6.38 & \\
\hline $\mathrm{AA}$ & 0 & 0.00 & 0 & 0 & \\
\hline $\mathrm{G}$ & 76 & 100 & 182 & 96.81 & \multirow[b]{2}{*}{ NS } \\
\hline $\mathrm{A}$ & 0 & 0 & 6 & 3.19 & \\
\hline \multicolumn{6}{|l|}{ rs8192720 } \\
\hline CC & 37 & 97.37 & 94 & 100 & \multirow{3}{*}{ NS } \\
\hline $\mathrm{CT}$ & 1 & 2.63 & 0 & 0 & \\
\hline $\mathrm{TT}$ & 0 & 0.00 & 0 & 0 & \\
\hline $\mathrm{C}$ & 75 & 98.68 & 188 & 100.00 & \multirow{2}{*}{ NS } \\
\hline $\mathrm{T}$ & 1 & 1.32 & 0 & 0.00 & \\
\hline \multicolumn{6}{|l|}{ rs1137115 } \\
\hline GG & 27 & 71.05 & 69 & 73.4 & \multirow{3}{*}{ NS } \\
\hline GA & 10 & 26.32 & 23 & 24.47 & \\
\hline AA & 1 & 2.63 & 2 & 2.13 & \\
\hline G & 64 & 84.21 & 161 & 85.64 & \multirow{2}{*}{ NS } \\
\hline A & 12 & 15.79 & 27 & 14.36 & \\
\hline \multicolumn{6}{|l|}{ rs2431413 } \\
\hline $\mathrm{TT}$ & 38 & 100.00 & 69 & 73.4 & \multirow{3}{*}{ NS } \\
\hline $\mathrm{TC}$ & 0 & 0.00 & 22 & 23.4 & \\
\hline $\mathrm{CC}$ & 0 & 0.00 & 3 & 3.19 & \\
\hline $\mathrm{T}$ & 76 & 100.00 & 160 & 85.11 & \multirow{2}{*}{ NS } \\
\hline $\mathrm{C}$ & 0 & 0.00 & 28 & 14.89 & \\
\hline \multicolumn{6}{|l|}{ rs5031017 } \\
\hline GG & 19 & 50.00 & 43 & 45.74 & \multirow{3}{*}{ NS } \\
\hline GT & 13 & 34.21 & 35 & 37.23 & \\
\hline $\mathrm{TT}$ & 6 & 15.79 & 16 & 17.02 & \\
\hline $\mathrm{G}$ & 51 & 67.11 & 121 & 64.36 & \multirow[b]{2}{*}{ NS } \\
\hline $\mathrm{T}$ & 25 & 32.89 & 67 & 35.64 & \\
\hline
\end{tabular}

* $p$-Pearson's $\chi^{2}$. GF: genotype frequency, AF: allele frequency, NS: non-significant.

We evaluated seventeen SNPs in five genes (NRXN1, DRD4, HTR2A, CHRNA3, CHRNA5, and near to CYP2A6) related to nicotine addiction; data are shown in Table 5. In NRXN1 there were differences in three SNPs, rs10189159 $(p=0.0004)$ had higher genotype frequency TT in e-Dec group ( 0.84 vs. 0.46$)$; instead, TC ( 0.47 vs. 0.13$)$ and CC ( 0.05 vs. 0.02$)$ genotype frequencies were higher in the on-site group; the rs985919 $(p=0.0333)$ had higher genotype frequency TT in e-Dec group ( 0.55 vs. 0.30 ) but genotype (TG) had minor frequency ( 0.36 vs. 0.57$)$ and same for genotype GG (0.09 vs. 0.29$)$. The rs1882296 had statistical significance differences $(p=0.0348)$, the AA genotype had a higher frequency in the e-Dec group (0.68 vs. 0.47) and the genotypes AG and GG had higher frequencies in on-site group ( 0.45 vs. 0.28 and 0.06 vs. 0.02 , respectively). Two SNPs in CHRNA3 had differences statistically significant in genotype frequencies, $\operatorname{rs} 1317286(p=0.0311)$ AA genotype frequency was higher in e-Dec group ( 0.73 vs. 0.52$)$ and heterozygous AG genotype was higher in the on-site group ( 0.42 vs. 0.23$)$, minor allele GG had the same tendency $(0.05$ vs. 0.02$)$; rs615470 $(p=0.0355)$ had differences among genotype frequencies 
in groups where TT genotype frequency was higher in the e-Dec group (0.81 vs. 0.60$)$ and both TC ( 0.35 vs. 0.15$)$ and CC (0.04 vs. 0.02$)$ genotypes were higher in the on-site group. Two SNPs in CHRNA5 had statistically significant differences in genotype frequencies, rs17408276 ( $p=0.0161)$ TT genotype frequency was higher in e-Dec group ( 0.81 vs. 0.56$)$ and the heterozygous TC genotype was higher in the on-site group (0.41 vs. 0.15$)$, minor allele CC genotype was similar in both groups $(0.02$ vs. 0.02$)$; rs951266 $(p<0.0001)$ had differences among genotype frequencies in groups where CC genotype frequency was higher in e-Dec group (0.94 vs. 0.54$)$ and both CT (0.40 vs. 0.05$)$ and TT (0.05 vs. 0.00$)$ genotypes were higher in on-site group.

Table 5. Genotype and allele frequencies in both groups included in this study in NRXN1, DRD4, HTR2A, CHRNA3, CHRNA5, and near CYP2A6.

\begin{tabular}{|c|c|c|c|c|c|}
\hline \multirow{2}{*}{$\begin{array}{c}\text { Gene/SNP } \\
\text { Genotype/Allele }\end{array}$} & \multicolumn{2}{|c|}{ e-Dec } & \multicolumn{2}{|c|}{ On-Site } & \multirow{2}{*}{$p^{*}$} \\
\hline & $n=38$ & GF/AF $\%$ & $n=94$ & GF/AF $\%$ & \\
\hline \multirow{2}{*}{\multicolumn{6}{|c|}{ NRXN1 }} \\
\hline & & & & & \\
\hline TT & 32 & 84.21 & 44 & 46.8 & \multirow{3}{*}{0.0004} \\
\hline TC & 5 & 13.16 & 45 & 47.87 & \\
\hline CC & 1 & 2.63 & 5 & 5.32 & \\
\hline $\mathrm{T}$ & 69 & 90.79 & 133 & 70.74 & \multirow[b]{2}{*}{0.0009} \\
\hline C & 7 & 9.21 & 55 & 29.26 & \\
\hline $\begin{array}{c}\text { rs985919 } \\
\text { TT }\end{array}$ & 21 & 55.26 & 29 & 30.85 & \multirow{3}{*}{0.0333} \\
\hline TG & 14 & 36.84 & 54 & 57.44 & \\
\hline GG & 3 & 7.89 & 11 & 11.7 & \\
\hline $\mathrm{T}$ & 56 & 73.68 & 112 & 59.57 & \multirow{2}{*}{0.0437} \\
\hline $\begin{array}{c}\mathrm{G} \\
\mathrm{rs} 10865246\end{array}$ & 20 & 26.32 & 76 & 40.43 & \\
\hline AA & 21 & 55.26 & 38 & 40.42 & \multirow{3}{*}{0.0621} \\
\hline $\mathrm{AC}$ & 15 & 39.47 & 42 & 44.68 & \\
\hline $\begin{array}{c}\mathrm{CC} \\
\mathrm{A}\end{array}$ & 2 & $\begin{array}{l}5.26 \\
7500\end{array}$ & 14 & $\begin{array}{l}14.89 \\
6277\end{array}$ & \\
\hline $\begin{array}{l}\text { A } \\
\text { C }\end{array}$ & 19 & $\begin{array}{l}7.00 \\
25.00\end{array}$ & $\begin{array}{l}118 \\
70\end{array}$ & 37.23 & \multirow[t]{2}{*}{0.0783} \\
\hline rs1882296 & & & & & \\
\hline AA & 26 & 68.42 & 45 & 47.87 & \multirow{3}{*}{0.0348} \\
\hline AG & 11 & 28.95 & 43 & 45.74 & \\
\hline GG & 1 & 2.63 & 6 & 6.38 & \\
\hline A & 63 & 82.89 & 133 & 70.74 & \multirow{2}{*}{0.0589} \\
\hline G & 13 & 17.11 & 55 & 29.26 & \\
\hline \multirow{2}{*}{\multicolumn{6}{|c|}{ DRD4 }} \\
\hline & & & & & \\
\hline TT & 19 & 50 & 43 & 45.74 & \multirow{3}{*}{0.2936} \\
\hline TC & 17 & 44.74 & 37 & 39.36 & \\
\hline CC & 2 & 5.26 & 14 & 14.89 & \\
\hline $\mathrm{T}$ & 55 & 72.37 & 123 & 65.43 & \multirow{2}{*}{$<0.0001$} \\
\hline C & 21 & 27.63 & 65 & 34.57 & \\
\hline \multirow{2}{*}{\multicolumn{6}{|c|}{ HTR2A }} \\
\hline & & & & & \\
\hline GG & 19 & 50 & 35 & 37.23 & \multirow{3}{*}{0.3103} \\
\hline GA & 14 & 36.84 & 46 & 48.93 & \\
\hline AA & 5 & 13.16 & 13 & 13.82 & \\
\hline $\mathrm{G}$ & 52 & 68.42 & 116 & 61.70 & \multirow{2}{*}{0.3750} \\
\hline$\underset{\text { rs6313 }}{\mathrm{A}}$ & 24 & 31.58 & 72 & 38.30 & \\
\hline CC & 22 & 57.89 & 77 & 81.91 & \multirow{3}{*}{0.0772} \\
\hline CT & 14 & 36.84 & 9 & 9.57 & \\
\hline TT & 2 & 5.26 & 8 & 8.51 & \\
\hline C & 58 & 76.32 & 163 & 86.70 & \multirow{2}{*}{0.0594} \\
\hline $\mathrm{T}$ & 18 & 23.68 & 25 & 13.30 & \\
\hline
\end{tabular}


Table 5. Cont.

\begin{tabular}{|c|c|c|c|c|c|}
\hline \multirow{2}{*}{$\begin{array}{c}\text { Gene/SNP } \\
\text { Genotype/Allele }\end{array}$} & \multicolumn{2}{|c|}{ e-Dec } & \multicolumn{2}{|c|}{ On-Site } & \multirow{2}{*}{$p^{*}$} \\
\hline & $n=38$ & GF/AF \% & $n=94$ & GF/AF \% & \\
\hline \multirow{2}{*}{\multicolumn{6}{|c|}{ CHRNA3 }} \\
\hline rs12914385 & & & & & \\
\hline CC & 23 & 60.53 & 49 & 52.12 & \multirow{3}{*}{0.6203} \\
\hline CT & 12 & 31.58 & 40 & 42.55 & \\
\hline TT & 3 & 7.89 & 5 & 5.32 & \\
\hline C & 58 & 76.32 & 138 & 73.40 & \multirow{3}{*}{0.7380} \\
\hline $\mathrm{T}$ & 18 & 23.68 & 50 & 26.60 & \\
\hline rs1317286 & & & & & \\
\hline AA & 28 & 73.68 & 49 & 52.12 & \multirow{3}{*}{0.0311} \\
\hline $\mathrm{AG}$ & 9 & 23.68 & 40 & 42.55 & \\
\hline GG & 1 & 2.63 & 5 & 5.32 & \\
\hline A & 63 & 82.89 & 138 & 73.40 & \multirow{3}{*}{0.1390} \\
\hline G & 13 & 17.11 & 50 & 26.60 & \\
\hline rs6495307 & & & & & \\
\hline CC & 29 & 76.32 & 53 & 56.38 & \multirow{3}{*}{0.0903} \\
\hline CT & 7 & 18.42 & 37 & 39.36 & \\
\hline TT & 2 & 5.26 & 4 & 4.25 & \\
\hline $\mathrm{C}$ & 65 & 85.53 & 143 & 76.06 & \multirow{3}{*}{0.1240} \\
\hline $\mathrm{T}$ & 11 & 14.47 & 45 & 23.94 & \\
\hline rs615470 & & & & & \\
\hline TT & 31 & 81.58 & 57 & 60.63 & \multirow{3}{*}{0.0355} \\
\hline TC & 6 & 15.79 & 33 & 35.1 & \\
\hline $\mathrm{CC}$ & 1 & 2.63 & 4 & 4.25 & \\
\hline $\mathrm{T}$ & 68 & 89.47 & 147 & 78.19 & \multirow[b]{2}{*}{0.0499} \\
\hline $\mathrm{C}$ & 8 & 10.53 & 41 & 21.81 & \\
\hline \multicolumn{6}{|l|}{ CHRNA5 } \\
\hline rs16969968 & & & & & \\
\hline GG & 25 & 65.79 & 53 & 56.38 & \\
\hline GA & 11 & 28.95 & 35 & 37.23 & 0.3700 \\
\hline AA & 2 & 5.26 & 6 & 6.38 & \\
\hline G & 61 & 80.26 & 141 & 75.00 & \\
\hline A & 15 & 19.74 & 47 & 25.00 & 0.4510 \\
\hline rs17408276 & & & & & \\
\hline TT & 31 & 81.58 & 53 & 56.38 & \\
\hline TC & 6 & 15.79 & 39 & 41.48 & 0.0161 \\
\hline $\mathrm{CC}$ & 1 & 2.63 & 2 & 2.12 & \\
\hline $\mathrm{T}$ & 62 & 81.58 & 145 & 77.13 & \\
\hline C & 14 & 18.42 & 43 & 22.87 & 0.5280 \\
\hline rs951266 & & & & & \\
\hline CC & 36 & 94.74 & 51 & 54.25 & \\
\hline CT & 2 & 5.26 & 38 & 40.42 & $<0.0001$ \\
\hline TT & 0 & 0 & 5 & 5.31 & \\
\hline $\mathrm{C}$ & 74 & 97.37 & 140 & 74.47 & \\
\hline $\mathrm{T}$ & 2 & 2.63 & 48 & 25.53 & $<0.0001$ \\
\hline rs680244 & & & & & \\
\hline GG & 27 & 71.05 & 52 & 55.32 & \\
\hline GA & 10 & 26.32 & 37 & 39.36 & 0.1006 \\
\hline AA & 1 & 2.63 & 5 & 5.31 & \\
\hline G & 64 & 84.21 & 141 & 75.00 & \\
\hline A & 12 & 15.79 & 47 & 25.00 & 0.1430 \\
\hline rs17486278 & & & & & \\
\hline AA & 24 & 63.16 & 46 & 48.93 & \\
\hline AC & 11 & 28.95 & 43 & 45.74 & 0.3213 \\
\hline CC & 3 & 7.89 & 5 & 5.31 & \\
\hline A & 59 & 77.63 & 153 & 81.38 & \\
\hline $\mathrm{C}$ & 17 & 22.37 & 35 & 18.62 & 0.6000 \\
\hline $\begin{array}{l}\text { near to } C Y P 2 A 6 \\
\text { rs4105144 }\end{array}$ & & & & & \\
\hline TT & 26 & 68.42 & 62 & 65.95 & \\
\hline TC & 10 & 26.32 & 29 & 30.85 & 0.9709 \\
\hline CC & 2 & 5.26 & 3 & 3.19 & \\
\hline $\mathrm{T}$ & 62 & 81.58 & 153 & 81.38 & \\
\hline $\mathrm{C}$ & 14 & 18.42 & 35 & 18.62 & 0.9701 \\
\hline
\end{tabular}

* $p$-Pearson's $\chi^{2}$. GF: genotype frequency; AF: allele frequency. 


\subsection{Metabolizer Classification by CYP2A6 Alleles}

We classify individuals by metabolizer type according to the polymorphic SNPs in our sample (rs28399433, rs28399434, rs8192720, rs2431413, and rs5031017) by their effect reported in the literature. We determine that two or more minor alleles of these SNPs were a slow metabolizer, one minor allele was an intermediate metabolizer and none-minor alleles were a normal metabolizer. We excluded rs1137115 because it was a non-specific marker of decreased function alleles. With this classification we could evaluate normal metabolizers $(n=19)$, intermediate metabolizers $(n=39)$, and slow metabolizers $(n=48)$. Comparing tobacco-related variables in these groups, only cigarettes per day were different in this classification $(p=0.038)$, in which slow metabolizers were those who smoked slightly more (10 (9.5-15)) than intermediate (10 (8-14)) and normal (8 (5-11)) metabolizers.

Figure 1 shows how different $C Y P 2 A 6$ alleles can harbor more than one SNP from the selected panel. Subjects were categorized into groups by their CYP2A6 allele effect reported in the literature. CYP2A6 were grouped as loss-of-function $\left({ }^{*} 2,{ }^{*}\right.$, and $\left.{ }^{*} 10\right)$, decreased $\left({ }^{*}\right.$, ${ }^{*} 9,{ }^{*} 13, * 15,{ }^{*} 19,{ }^{*} 21$, and $\left.{ }^{*} 38\right)$ and normal function $\left({ }^{*} 1,{ }^{*} 8\right)$, the remaining alleles did not have information in the literature $\left({ }^{*} 18, * 36\right.$, and $\left.{ }^{*} 37\right)$. Once each subject had genotyped their corresponding alleles, their genotypes were grouped into the enzymatic activity by nicotine metabolite ratio, which is evaluated by the $3 \mathrm{HC} / \mathrm{COT}$ ratio [19] as normal (two normal function alleles), intermediate (one normal function allele and one decreased function allele) or slow metabolizers (one normal function allele and one loss-of-function allele; one decreased function allele and loss-of-function allele; two decreased function alleles; two loss-of-function alleles).

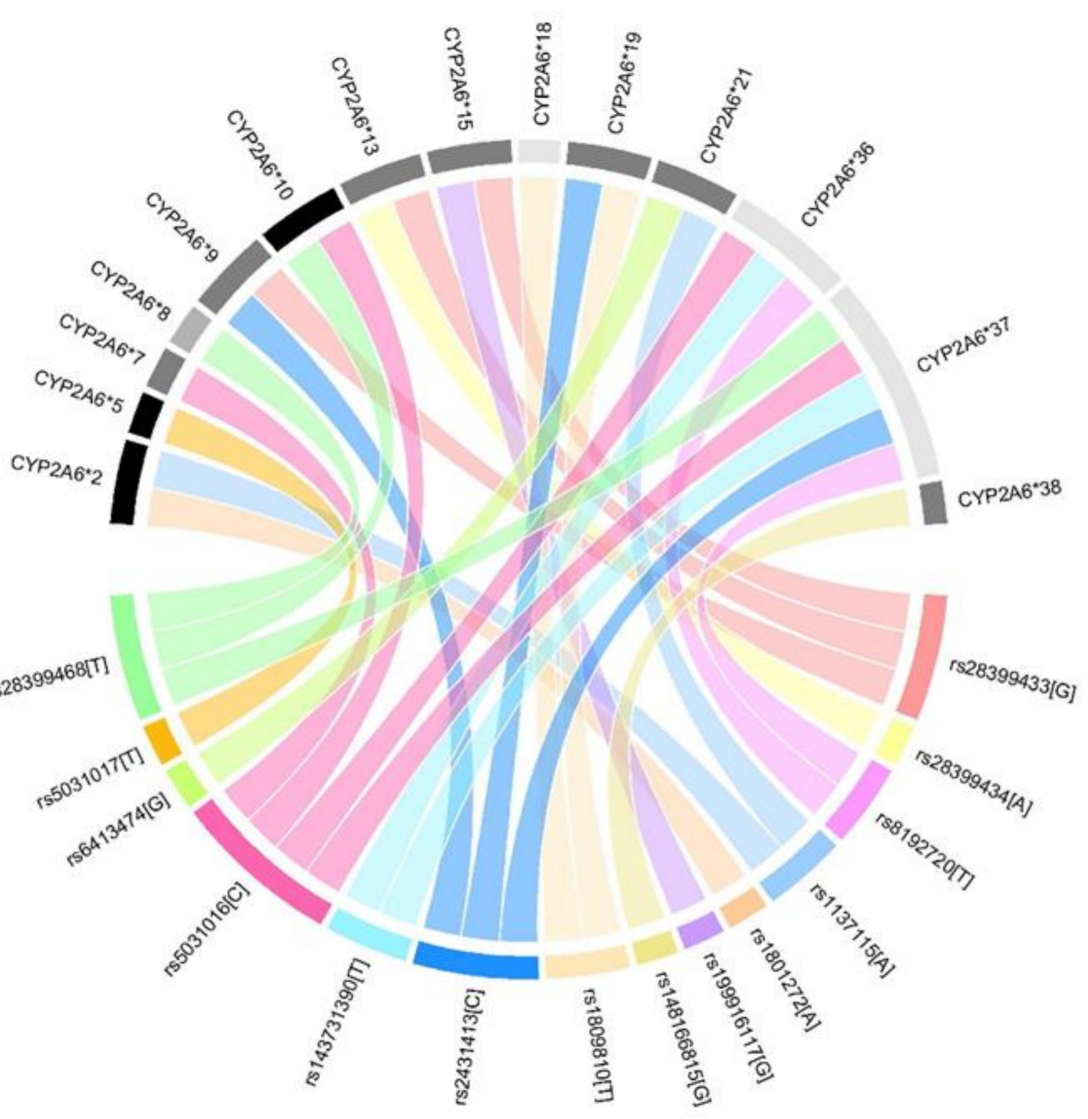

CYP2A6 allele function

Loss-of-function

Decreased function

Normal function

Unknown function

Figure 1. Circle plot for CYP2A6 alleles. SNPs assign CYP2A6 alleles and functions according to PharmVar Consortium. 


\subsection{Matched Sub-Analysis}

We matched e-Dec with on-site groups by age and gender to avoid confounding variables due to group differences in demographic variables. We keep the thirty-eight e-Dec and sixty-two on-site participants. Although these groups have similar age $(p=0.847)$ and gender proportions $(p=0.542)$, both groups have differences in tobacco smoking variables. e-Dec group smoked fewer cigarettes per day $(p=0.043)$, by fewer years $(p=0.014)$, and by fewer cumulative pack-years $(p=0.003)$. Interestingly, we obtained a marginal difference in the Fagerström Test for Nicotine Dependence (FTND) $(p=0.053)$ with higher values in the e-Dec group. In the rs28399433 genotype evaluation, this kept with differences among both groups $(p<0.0001)$, mainly in TT (0.78 vs. 0.29$)$ and TG $(0.18$ vs. 0.67$)$ genotype frequencies. The remaining SNPs lack statistically significant differences among their genotype frequencies.

\section{Discussion}

In the current study, we report for the first time CYP2A6 SNPs in Mexican mestizo smokers and their distribution among two different smoking cessation programs. Previously, we had reported other SNPs in smoking-related genes as NRXN1, DRD4, HTR2A, CHRNA3, CHRNA5, and CYP2A6 in Mexican mestizo, focused on smokers enrolled in a smoking cessation program, mostly with high tobacco consumption and nicotine dependence $[15,16,20]$. Here, we describe genotype frequencies of SNPs in smoking-related genes on an innovative based in text messages program, which enrolled smokers from the general population.

In our study groups, tobacco-related variables (cigarettes per day, years of smoking, and pack-years) do not reflect nicotine dependence assessed by FTND; despite individuals in the e-Dec group smoking less, they had a higher score in the questionnaire. Although the FTND application has been discussed in the Mexican Mestizo population [21], it is the most-employed test to assess nicotine dependence in the scientific literature.

Despite their effect on the enzyme activity, selected CYP2A6 SNPs, according to assignation by the Pharmacogene Variation Consortium [14], only six were polymorphic; that is, no minor allele was identified in nine SNPs potentially related to alteration in the ability to metabolize nicotine. This is probably due to miscegenation in the Mexican mestizo population in which the ancestral Amerindian component reaches 55\% [22], and SNPs reported as CYP2A6 activity-related are mainly evaluated in Caucasian populations. We found differences among two cessation programs in two SNPs, rs28399433 and rs2431413; previously, the rs28399433 showed reduced apparent oral efavirenz clearance [23]; regarding nicotine metabolism, women and normal nicotine metabolizers (harboring the common allele) may benefit more from varenicline over nicotine replacement therapy [24]. Previously, our workgroup identified an association with a younger age at onset smoking [16], smoking status, age of onset, and psychological dependence in Mexican mestizo families [15,25].

With regard to single nucleotide variants analyzed in the genes related to those involved in the dopaminergic and serotonergic pathways, we identified differences in the genotype frequency among both groups in three SNPs in NRXN1 gene, the rs10189159, rs985919, and rs1882296; the latter revealed in an in silico analysis that rs1882296/T had a high level of homology with Hsa-miR-6740-5p, which encodes a putative miRNA that targets glutamate receptor subunits (GRIA2, GRID2) and GABA receptor subunits (GABRG1, GABRA4, GABRB2). In contrast, rs1882296/C had a high homology level with Hsa-miR6866-5p, which encodes a different miRNA that targets GRID2 and GABRB2 [25], proposing new hypotheses regarding the putative roles of miRNAs that influence the GABAergic and glutamatergic pathways in smoking addiction. Otherwise, two SNPs in CHRNA3 had statistically significant differences in genotype frequencies, rs1317286 and rs615470. Using a family-based association test, Li and colleagues found a nominal association rs1317286 and rs8040868 in CHRNA3 with nicotine dependence in the African Americans and combined with European American samples. However, this association was not significant after correction for multiple testing [26]. Finally, the rs17408276 and rs951266 in the CHRNA5 
gene were found in different genotype frequencies among groups; the rs17408276 variant moderates nicotine deprivation, a neural index of cognitive control [27], and was associated with increased lung cancer risk in African Americans [28]. In comparison, rs951266 has been reported to be associated with schizophrenia and bipolar disorder [29].

This study is not exempt from limitations. The first one refers to the sampling method. In this study, only patients' samples from the previously published e-Decídete! program were included, and maybe the most important is the reduced sample size compared to others; current results also only showed baseline data from two different interventions. Later studies including the risk of relapse after the end of treatment are desirable. In addition, no other potential confounders (as marital or socio-economic status) were recorded among the participants. Besides, although the Fagerström test is a suitable instrument to evaluate nicotine addiction, each consumer has their style for smoking (puff number, volume, interval, and duration); we were not able to analyze blood or urine cotinine levels in the on-site group to ascertain the self-reported cigarettes per day smoked by the subjects.

The main strength is the comparison of two different interventions for quitting smoking. Moreover, this is the first time a mobile-based smoking cessation intervention has been compared in terms of smokers' genetic characteristics. This collaborative research establishes the basis for future investigations where smoking cessation interventions can be aborded from a genetic perspective. Among the practical implications of this study is the characterization of the smoking behavior of subjects attending two different smoking cessation interventions. Future research efforts should focus on fully characterizing the functional genetic variation and identifying the impact of these functional variants on smoking and treatment outcomes.

\section{Conclusions}

Tobacco smoking subjects attending a mobile smoking cessation intervention smoke fewer cigarettes per day by fewer years and fewer cumulative pack-years. There are differences in the genotype frequencies of SNPs in genes related to nicotine metabolism (CYP2A6) and nicotine dependence (NRXN1, DRD4, CHRNA3-CHRNA5). Slow metabolizers smoke more cigarettes per day than intermediate and normal metabolizers.

Author Contributions: Conceptualization, A.P.C., L.M.R.-S., E.F.E. and R.F.-V.; data curation, R.F.V.; formal analysis, G.P.-R. and R.F.-V.; funding acquisition, A.P.C., L.M.R.-S., E.F.E. and R.F.-V.; investigation, G.P.-R., L.A.L.-F. and R.R.-B.; methodology, G.P.-R., L.A.L.-F., F.C.-B. and M.R.; project administration, A.P.C., F.C.-B., L.M.R.-S., M.R., E.F.E. and R.F.-V.; resources, A.P.C., F.C.-B. and M.R.; software, G.P.-R., L.A.L.-F. and R.R.-B.; supervision, A.P.C., L.M.R.-S., E.F.E., R.R.-B. and R.F.-V.; validation, F.C.-B.; visualization, A.P.C., L.M.R.-S., M.R., E.F.E. and R.R.-B.; writing-original draft, G.P.-R., L.A.L.-F. and R.F.-V.; writing-review and editing, G.P.-R., L.A.L.-F. and R.F.-V. All authors have read and agreed to the published version of the manuscript.

Funding: This research received no external funding.

Institutional Review Board Statement: The study was conducted according to the Declaration of Helsinki's guidelines and approved by the Institutional Committees for Research, Ethics in Research, and Biosecurity of the Instituto Nacional de Enfermedades Respiratorias Ismael Cosio Villegas approved this study (approval code numbers: B07-17 and B15-16 on 14 February 2016).

Informed Consent Statement: Informed consent was obtained from all subjects involved in the study.

Data Availability Statement: All data generated for this study are included in this article and its supplementary information file.

Acknowledgments: The authors acknowledge the support received from physicians, nurses, technicians, and administrative personnel from the INER and INSP to acquire data and samples of the study participants.

Conflicts of Interest: The authors declare no conflict of interest. 


\section{References}

1. [ENCODAT] Instituto Nacional de Psiquiatría Ramón de la Fuente Muñiz; Instituto Nacional de Salud Pública, Comisión Nacional Contra las Adicciones, Secretaría de Salud. Encuesta Nacional de Consumo de Drogas, Alcohol y Tabaco 2016-2017: Reporte de Tabaco; Reynales-Shigematsu, L.M., Zavala-Arciniega, L., Paz-Ballesteros, W.C., Gutiérrez-Torres, D.S., García-Buendía, J.C., Rodriguez-Andrade, M.A., Gutiérrez-Reyes, J., Franco-Núñez, A., Romero-Martínez, M., y Mendoza-Alvarado, L., Eds.; INPRFM: Ciudad de México, México, 2017.

2. Organización Panamericana de la Salud; Instituto Nacional de Salud Pública. Encuesta Global de Tabaquismo en Adultos; INSP/OPS: Cuernavaca, México, 2017.

3. Ducci, F.; Goldman, D. The Genetic Basis of Addictive Disorders. Psychiatry Clin. N. Am. 2012, 35, 495-519. [CrossRef] [PubMed]

4. Tyndale, R.F.; Zhu, A.Z.; George, T.P.; Cinciripini, P.; Hawk, L.W., Jr.; Schnoll, R.A.; Swan, G.E.; Benowitz, N.L.; Heitjan, D.F.; Lerman, C.; et al. Lack of associations of CHRNA5-A3-B4 genetic variants with smoking cessation treatment outcomes in Caucasian smokers despite associations with baseline smoking. PLoS ONE 2015, 10, e0128109. [CrossRef] [PubMed]

5. Benowitz, N.L.; Hukkanen, J.; Jacob, P., 3rd. Nicotine chemistry metabolism kinetics and biomarkers. In Nicotine Psychopharmacology; Handbook of Experimental Pharmacology Series; Springer: Berlin/Heidelberg, Germany, 2009; pp. 29-60.

6. Tyndale, R.F.; Mwenifumbo, J.C. Molecular genetics of nicotine metabolism. In Nicotine Psychopharmacology; Handbook of Experimental Pharmacology Series; Springer: Berlin/Heidelberg, Germany, 2009; Volume 192, pp. $235-259$.

7. Al Koudsi, N.; Hoffmann, E.B.; Assadzadeh, A.; Tyndale, R.F. Hepatic CYP2A6 levels and nicotine metabolism: Impact of genetic, physiological, environmental, and epigenetic factors. Eur. J. Clin. Pharmacol. 2010, 66, 239-251. [CrossRef] [PubMed]

8. Bloom, A.J.; Harari, O.; Martinez, M.; Zhang, X.; McDonald, S.A.; Murphy, S.E.; Goate, A. A compensatory effect upon splicing results in normal function of the CYP2A6*14 allele. Pharmacogenet. Genom. 2013, 23, 107-116. [CrossRef] [PubMed]

9. Tanner, J.A.; Tyndale, R.F. Variation in CYP2A6 activity and personalized medicine. J. Pers. Med. 2017, 7, 18. [CrossRef] [PubMed]

10. Chen, L.-S.; Saccone, N.L.; Culverhouse, R.C.; Bracci, P.M.; Chen, C.H.; Dueker, N.; Han, Y.; Huang, H.; Jin, G.; Kohno, T.; et al. Smoking and Genetic Risk Variation Across Populations of European, Asian, and African American Ancestry-A Meta-Analysis of Chromosome 15q25. Genet. Epidemiol. 2012, 36, 340-351. [CrossRef] [PubMed]

11. Saccone, N.L.; Culverhouse, R.C.; Schwantes-An, T.-H.; Cannon, D.S.; Chen, X.; Cichon, S.; Giegling, I.; Han, S.; Han, Y.; Keskitalo-Vuokko, K.; et al. Multiple independent loci at chromosome 15q25.1 affect smoking quantity: A meta-analysis and comparison with lung cancer and COPD. PLoS Genet. 2010, 6, e1001053. [CrossRef] [PubMed]

12. Wang, J.C.; Cruchaga, C.; Saccone, N.L.; Bertelsen, S.; Liu, P.; Budde, J.; Duan, W.; Fox, L.; Grucza, R.A.; Kern, J.; et al. Risk for nicotine dependence and lung cancer is conferred by mRNA expression levels and amino acid change in CHRNA5. Hum. Mol. Genet. 2009, 18, 3125-3135. [CrossRef] [PubMed]

13. Bierut, L.J.; Tyndale, R.F. Preparing the Way: Exploiting Genomic Medicine to Stop Smoking. Trends Mol. Med. 2018, 24, 187-196. [CrossRef]

14. Gaedigk, A.; Ingelman-Sundberg, M.; Miller, N.A.; Leeder, J.S.; Whirl-Carrillo, M.; Klein, T.E. The Pharmacogene Variation (PharmVar) Consortium: Incorporation of the Human Cytochrome P450 (CYP) Allele Nomenclature Database. Clin. Pharmacol. Ther. 2018, 103, 399-401. [CrossRef]

15. Pérez-Rubio, G.; Perez-Rodriguez, M.E.; Fernández-López, J.C.; Ramirez-Venegas, A.; Garcia-Colunga, J.; Avila-Moreno, F.; Camarena, A.; Sansores, R.H.; Falfan-Valencia, R. SNPs in NRXN1 and CHRNA5 are associated to smoking and regulation of GABAergic and glutamatergic pathways. Pharmacogenomics 2016, 17, 1145-1158. [CrossRef]

16. Pérez-Rubio, G.; López-Flores, L.A.; Ramírez-Venegas, A.; Noé-Díaz, V.; García-Gómez, L.; Ambrocio-Ortiz, E.; Sánchez-Romero, C.; Hernández-Zenteno, R.D.J.; Sansores, R.H.; Falfán-Valencia, R. Genetic polymorphisms in CYP2A6 are associated with a risk of cigarette smoking and predispose to smoking at younger ages. Gene 2017, 628, 205-210. [CrossRef]

17. Public Health Information Department of the General Public Health Administration of the Health Consulate. EPIDAT, Program for Epidemiological Analysis of Tabulated Data, Version 3.1.; WHO: Geneva, Switzerland, 2006.

18. Cupertino, A.P.; Cartujano-Barrera, F.; Ramírez, M.; Rodríguez-Bolaños, R.; Thrasher, J.F.; Pérez-Rubio, G.; Falfán-Valencia, R.; Ellerbeck, E.F.; Reynales-Shigematsu, L.M. A mobile smoking cessation intervention for Mexico (vive sin tabaco ... ¡decídete!): Single-arm pilot study. J. Med. Internet Res. 2019, 7, e12482. [CrossRef]

19. Dempsey, D.; Tutka, P.; Iii, P.J.P.; Allen, F.; Schoedel, K.; Tyndale, R.; Benowitz, N.L. Nicotine metabolite ratio as an index of cytochrome P450 2A6 metabolic activity. Clin. Pharmacol. Ther. 2004, 76, 64-72. [CrossRef]

20. Pérez-Rubio, G.; Ramírez-Venegas, A.; Díaz, V.N.; Gómez, L.G.; Fabián, K.E.; Carmona, S.G.; López-Flores, L.A.; Ambrocio-Ortiz, E.; Romero, R.C.; Alcantar-Ayala, N.; et al. Polymorphisms in HTR2A and DRD4 Predispose to Smoking and Smoking Quantity. PLOS ONE 2017, 12, e0170019.

21. Moreno-Coutiño, A.; Villalobos-Gallegos, L. Psychometric Properties of the Fagerström Test for Nicotine Dependence in a Sample of Mexican Smokers. J. Addict. Nurs. 2017, 28, 27-33. [CrossRef]

22. Pérez-Rubio, G.; Silva-Zolezzi, I.; Fernández-López, J.C.; Camarena, Á.; Velázquez-Uncal, M.; Morales-Mandujano, F.; HernándezZenteno, R.D.J.; Flores-Trujillo, F.; Sánchez-Romero, C.; Velázquez-Montero, A.; et al. Genetic Variants in IL6R and ADAM19 are Associated with COPD Severity in a Mexican Mestizo Population. COPD J. Chronic Obstr. Pulm. Dis. 2016, 13, 610-615. [CrossRef]

23. Vujkovic, M.; Bellamy, S.L.; Zuppa, A.F.; Gastonguay, M.R.; Moorthy, G.S.; Ratshaa, B.; Han, X.; Steenhoff, A.P.; Mosepele, M.; Strom, B.L.; et al. Polymorphisms in cytochrome P450 are associated with extensive efavirenz pharmacokinetics and CNS toxicities in an HIV cohort in Botswana. Pharmacogenom. J. 2018, 18, 678-688. [CrossRef] 
24. Glatard, A.; Dobrinas, M.; Gholamrezaee, M.; Lubomirov, R.; Cornuz, J.; Csajka, C.; Eap, C.B. Association of nicotine metabolism and sex with relapse following varenicline and nicotine replacement therapy. Exp. Clin. Psychopharmacol. 2017, 25, 353-362. [CrossRef]

25. Svyryd, Y.; Ramírez-Venegas, A.; Hernandez, B.S.; Aguayo-Gómez, A.; Luna-Muñoz, L.; Arteaga-Vazquez, J.; Regalado-Piñeda, J.; Mutchinick, O.M. Genetic Risk Determinants for Cigarette Smoking Dependence in Mexican Mestizo Families. Nicotine Tob. Res. 2016, 18, 620-625. [CrossRef]

26. Li, M.D.; Xu, Q.; Lou, X.-Y.; Payne, T.J.; Niu, T.; Ma, J.Z. Association and interaction analysis of variants in CHRNA5/CHRNA3/ CHRNB4 gene cluster with nicotine dependence in African and European Americans. Am. J. Med. Genet. B. Neuropsychiatr. Genet. 2010, 153B, 745-756.

27. Evans, D.E.; MacQueen, D.A.; Jentink, K.G.; Park, J.Y.; Lin, H.-Y.; Drobes, D.J. CHRNA5 variants moderate the effect of nicotine deprivation on a neural index of cognitive control. Genes. Brain. Behav. 2014, 13, 626-632. [CrossRef] [PubMed]

28. Hansen, H.M.; Xiao, Y.; Rice, T.; Bracci, P.M.; Wrensch, M.R.; Sison, J.D.; Chang, J.S.; Smirnov, I.V.; Patoka, J.; Seldin, M.F.; et al. Fine mapping of chromosome 15q25.1 lung cancer susceptibility in African-Americans. Hum. Mol. Genet. 2010, 19, 3652-3661. [CrossRef] [PubMed]

29. Jackson, K.J.; Fanous, A.H.; Chen, J.; Kendler, K.S.; Chen, X. Variants in the $15 \mathrm{q} 25$ gene cluster are associated with risk for schizophrenia and bipolar disorder. Psychiatr. Genet. 2013, 23, 20-28. [CrossRef] [PubMed] 